\title{
Elements of a Spatial Web
}

\author{
Christian S. Jensen \\ Department of Computer Science, Aarhus University, Denmark \\ csj@cs.au.dk
}

\begin{abstract}
Driven by factors such as the increasingly mobile use of the web and the proliferation of geo-positioning technologies, the web is rapidly acquiring a spatial aspect. Specifically, content and users are being geo-tagged, and services are being developed that exploit these tags. The research community is hard at work inventing means of efficiently supporting new spatial query functionality.

Points of interest with a web presence, called spatial web objects, have a location as well as a textual description. Spatio-textual queries return such objects that are near a location argument and are relevant to a text argument. An important element in enabling such queries is to be able to rank spatial web objects. Another is to be able to determine the relevance of an object to a query. Yet another is to enable the efficient processing of such queries. The talk covers recent results on spatial web object ranking and spatio-textual querying obtained by the speaker and his colleagues.
\end{abstract}

